\title{
In vitro hydroxyapatite formation of a TTCP and DCPA based dentine desensitiser
}

Tomas Duminis, Saroash Shahid, Robert Hill, David Gillam, Alessia D’Onofrio

This manuscript has been withdrawn by the authors as it was submitted and made public without the full consent of all the authors. Therefore, the authors do not wish this work to be cited as reference for the project. If you have any questions, please contact the corresponding author. 